\title{
A Conservation Law Treatment of Nonlinear KdV Hierarchies
}

\author{
Sameerah Jamal $(D)$ and Rivoningo Maphanga \\ School of Mathematics, University of the Witwatersrand, Johannesburg, South Africa \\ Correspondence should be addressed to Sameerah Jamal; sameerah.jamal@wits.ac.za
}

Received 3 October 2021; Accepted 29 December 2021; Published 18 January 2022

Academic Editor: Naihuan Jing

Copyright (C) 2022 Sameerah Jamal and Rivoningo Maphanga. This is an open access article distributed under the Creative Commons Attribution License, which permits unrestricted use, distribution, and reproduction in any medium, provided the original work is properly cited.

We study the hierarchy commonly defined as an infinite sequence of partial differential equations which begins with the Korteweg-de Vries equation and its modified version. An important feature of the hierarchy is its highly nonlinear property. In this regard, obtaining solutions for the members of the hierarchy poses a great problem. In this paper, we propose a method to allow for the construction of solutions to the full hierarchy. Our approach involves a recursion operator in the conservation law of the hierarchy. The efficiency of the method is demonstrated by selected examples. In certain cases, we obtain snoidal solutions.

\section{Introduction}

The Korteweg-de Vries (KdV) equation,

$$
u_{t}=u_{x x x}-6 u u_{x}
$$

attributed to the Dutch mathematicians Diederik Korteweg and Gustav de Vries (1895), is a mathematical model of waves on shallow water surfaces whose origin has a long history. To this day, the KdV equation is still considered as one of the most important nonlinear partial differential equations as it has a wide variety of applications [1]. It was originally derived to model the propagation of weakly dispersive nonlinear water waves and serve as a model equation for any physical system in consideration [2]. In most contexts, $u(x, t)$ is the function that denotes the elongation of the wave at space $x$ and time $t$ [3].

$\mathrm{KdV}$ equation (1) naturally extends to an infinite sequence of integrable nonlinear partial differential equations of solitonic characters [4] and can be considered as the initiator of the KdV hierarchy, denoted by

$$
u_{t}=\left(D_{x}^{2}-4 u-2 u_{x} D_{x}^{-1}\right)^{n} u_{x}, n=1,2, \ldots,
$$

where $D_{x}$ denotes the total derivative and $D_{x}^{-1}$ denotes the integral with respect to $x$. The second member of the hierarchy is given when $n=2$ in (2), viz.

$$
u_{t}=u_{x x x x x}-20 u_{x} u_{x x}-10 u u_{x x x}+30 u^{2} u_{x} .
$$

Note that, for higher order $n$, the equations become increasingly nonlinear and higher order in derivatives.

If the nonlinear term $u u_{x}$ in equation (1) is replaced by $u^{m} u_{x}$, then the most important case other than when $m=1$, is when $m=2$. This yields the so-called modified $\mathrm{KdV}$ $(\mathrm{mKdV})$ equation, given by $[5,6]$

$$
u_{t}=u_{x x x}-6 u^{2} u_{x}
$$

which may also be connected to the mKdV hierarchy:

$$
v_{t}=\left(D_{x}^{2}-4 v^{2}-4 v_{x} D_{x}^{-1} v\right)^{n} v_{x .}
$$

Here, the case of $n=2$ gives the second member of the mKdV hierarchy:

$$
v_{t}=30 v_{x} v^{4}-10 v^{2} v_{x x x}-40 v v_{x} v_{x x}-10 v_{x}^{3}+v_{x x x x x} \text {. }
$$

Another famous hierarchy is the Burgers' hierarchy and its solutions $[7,8]$.

Completely integrable nonlinear equations, such as those of the KdV hierarchy, are endowed with many special mathematical properties. They are of interest due to their infinite conservation laws [9] and symmetries [10], bi- or triHamiltonian structures, their Painlevé property [11], Lax pairs [12], etc. The solving of such equations is deeply connected to the inverse scattering transform $[13,14]$ and 
Hirota's direct method [15]. The aforementioned importance of the KdV hierarchy has motivated this study.

The $\mathrm{mKdV}$ equations are related to the $\mathrm{KdV}$ equation through the Miura transformation, which maps the solutions of the $\mathrm{KdV}$ equations to the solutions of the $\mathrm{mKdV}$ equations [16].

The KdV hierarchies are an example of higher-order water wave models, which are of great significance, and they play crucial roles especially to study physical systems and the necessary material properties needed to manipulate waves in a desired manner [17].

In this paper, we propose a method to solve the full $\mathrm{KdV}$ hierarchy and extend the method to include the mKdV hierarchy. This is a novel proposal as, to the best of our knowledge, no such endeavour has appeared in the literature. The entire hierarchy is highly nonlinear and of higher order in derivatives, thereby posing an extremely challenging problem to solve. We formulate our method based on a transformation of variables (derived from the point symmetries of the hierarchy), effectively reducing the partial differential hierarchy into an ordinary differential hierarchy. The latter is connected to a transformed conservation law of the hierarchy, and the knowledge of this transformed conservation law forms a general approach to solving the hierarchy for all $n$.

This paper is organised as follows. In Section 2, we briefly present some theoretical considerations, and Section 3 discusses some general properties of the hierarchies. Section 4 contains the main results and a description of our method, and Section 5 elaborates on some applications of our method. Section 6 provides some alternate solutions.

\section{Preliminaries}

The procedure for determining point symmetries for an arbitrary system of equations is well known [18]. Consider $q$ unknown functions $u^{\alpha}$ which depend on $p$ independent variables $x^{i}$, i.e., $u=\left(u^{1}, \ldots, u^{q}\right)$ and $x=\left(x^{1}, \ldots, x^{p}\right)$, with indices $\alpha=1, \ldots, q$ and $i=1, \ldots, p$. Let

$$
G_{\alpha}\left(x, u^{(k)}\right)=0
$$

be a system of nonlinear differential equations, where $u^{(k)}$ represents the $k^{\text {th }}$ derivative of $u$ with respect to $x$. We consider the following symmetry:

$$
X=\xi^{i} \partial_{x^{i}}+\eta^{\alpha} \partial_{u^{\alpha}}
$$

given by

$$
X\left[G_{\alpha}\left(x, u^{(k)}\right)\right]=0, \text { when } G_{\alpha}\left(x, u^{(k)}\right)=0,
$$

where $X$ is extended to all derivatives appearing in the equation through an appropriate prolongation. A current $T=\left(T^{1}, \ldots, T^{n}\right)$ is conserved if it satisfies

$$
D_{i} T^{i}=0
$$

along the solutions of the given equation. Equation (10) is called a local conservation law.
Suppose that $X$ is a symmetry of system (7) and $T$ is a conserved vector of (7). Then, if $X$ and $T$ satisfy

$$
X\left(T^{i}\right)+T^{i} D_{j}\left(\xi^{j}\right)-T^{j} D_{j}\left(\xi^{i}\right)=0,
$$

the symmetry $X$ is said to be associated with $T$ [19]. Equation (11) is closely related to a Noether theorem, but in [19], it was proved that this result holds without the existence of Lagrangian. The transformation $u=v_{x}$ for the $\mathrm{KdV}$ equation enables the construction of a Lagrangian density [20] so that Noether's theorem may be applied for its conservation laws. However, we have opted to study the $\mathrm{KdV}$ equation in the absence of Lagrangian.

\section{Generalised Properties of the KdV and mKdV Hierarchy}

A standard calculation of the symmetries of equation (1), using condition (9), reveals that it has the following four Lie point symmetries:

$$
\begin{aligned}
& X_{1}=\partial_{t}, \\
& X_{2}=\partial_{x}, \\
& X_{3}=6 t \partial_{x}+\partial_{u}, \\
& X_{4}=3 t \partial_{t}-2 u \partial_{u}+x \partial_{x} .
\end{aligned}
$$

The second member of the hierarchy, with $n=2$, or equation (3) has the following three symmetries:

$$
X_{1}, X_{2}, X_{3}^{2}=5 t \partial_{t}+x \partial_{x}-2 u \partial_{u}
$$

If one repeats the Lie symmetry method for higher members, it is easy to see that hierarchy (2) possesses the Lie point symmetries

$$
X_{1}, X_{2}, X_{1}^{n}=(2 n+1) t \partial_{t}-2 u \partial_{u}+x \partial_{x}
$$

for $n \geq 2$ with Lie bracket relations $[A, B]=A B-B A$, given in Table 1.

A similar investigation of $\mathrm{mKdV}$ hierarchy (5) gives that $n=1$ has the following three symmetries:

$$
\begin{aligned}
& X_{1}, \\
& X_{2}, \\
& X_{6}=\frac{x}{3} \partial_{x}+t \partial_{t}-\frac{v}{3} \partial_{v},
\end{aligned}
$$

and $n=2$ has the symmetries

$$
\begin{aligned}
& X_{1}, \\
& X_{2}, \\
& X_{7}=\frac{x}{5} \partial_{x}+t \partial_{t}-\frac{v}{5} \partial_{v} .
\end{aligned}
$$

As before, if one repeats the Lie symmetry method for higher members, it is easy to see that hierarchy (5) possesses the Lie point symmetries 
TABLE 1: Lie brackets of symmetries (14).

\begin{tabular}{cccc}
\hline$[]$, & $X_{1}$ & $X_{2}$ & $X_{1}^{n}$ \\
\hline$X_{1}$ & 0 & 0 & $(2 n+1) X_{1}$ \\
$X_{2}$ & 0 & 0 & $X_{2}$ \\
$X_{1}^{n}$ & $-(2 n+1) X_{1}$ & $-X_{2}$ & 0 \\
\hline
\end{tabular}

$$
X_{1}=\partial_{t}, X_{2}=\partial_{x}, X_{2}^{n}=t \partial_{t}-\frac{v}{2 n+1} \partial_{v}+\frac{x}{2 n+1} \partial_{x},
$$

for $n \geq 2$ with Lie bracket relations in Table 2 .

As for the conservation laws of the above hierarchies, we notice several interesting properties. There exist many ways to compute conservation laws, and we opt for the multiplier approach [21].

Below are the cases of the KdV hierarchy when $n=1$ and $n=2$, where the conservation laws are $T_{i}=\left(T_{i}^{t}, T_{i}^{x}\right)$ where $i=1,2$, and 3 . Equation (1) has the following three conservation laws:

$$
\begin{aligned}
& T_{1}^{t}=u, \\
& T_{1}^{x}=3 u^{2}-u_{x x}, \\
& T_{2}^{t}=\frac{u^{2}}{2}, \\
& T_{2}^{x}=2 u^{3}+\frac{1}{2} u_{x}^{2}-u u_{x x},
\end{aligned}
$$

and finally,

$$
\begin{aligned}
& T_{3}^{t}=\frac{1}{2} t u^{2}-\frac{x}{6} u, \\
& T_{3}^{x}=-\frac{u_{x}}{6}-t u u_{x x}+2 t u^{3}-\frac{x}{2} u^{2}+\frac{t}{2} u_{x}^{2}+\frac{x}{6} u_{x x} .
\end{aligned}
$$

These conservation laws can also be found via Noether's theorem if the problem is reformulated to possess Lagrangian. The calculations are straightforward but tedious. Reports of these quantities or their equivalent appear in $[20,22]$, with more in [23].

Equation (3), i.e., $n=2$, has the following two conservation laws:

$$
\begin{aligned}
& T_{1}^{t}=u, \\
& T_{1}^{x}=-10 u^{3}+10 u u_{x x}+5 u_{x}^{2}-u_{x x x x}, \\
& T_{2}^{t}=\frac{u^{2}}{2}, \\
& T_{2}^{x}=-\frac{15}{2} u^{4}+10 u^{2} u_{x x}-u u_{x x x x}+u_{x} u_{x x x}-\frac{1}{2} u_{x x}^{2} .
\end{aligned}
$$

As for the mKdV equation, equation (4) has the following two conservation laws:
TABLE 2: Lie brackets of symmetries (17).

\begin{tabular}{cccc}
\hline$[]$, & $X_{1}$ & $X_{2}$ & $X_{2}^{n}$ \\
\hline$X_{1}$ & 0 & 0 & $\left(X_{1} / 2 n+1\right)$ \\
$X_{2}$ & 0 & 0 & $X_{2}$ \\
$X_{2}^{n}$ & $-\left(X_{1} / 2 n+1\right)$ & $-X_{2}$ & 0 \\
\hline
\end{tabular}

$$
\begin{aligned}
& T_{1}^{t}=\frac{1}{2} v^{2}, \\
& T_{1}^{x}=-v v_{x x}+\frac{1}{2} v_{x}^{2}+\frac{3}{2} v^{4}, \\
& T_{2}^{t}=v, \\
& T_{2}^{x}=2 v^{3}-v_{x x} .
\end{aligned}
$$

Similarly, equation (6), $n=2$, has the conservation laws

$$
\begin{aligned}
& T_{1}^{t}=\frac{1}{2} v^{2}, \\
& T_{1}^{x}=-5 v^{6}+10 v_{x x} v^{3}+5 v^{2} v_{x}^{2}-v v_{x x x x}+v_{x} v_{x x x}-\frac{1}{2} v_{x x}^{2}, \\
& T_{2}^{t}=v, \\
& T_{2}^{x}=-6 v^{5}+10 v^{2} v_{x x}+10 v v_{x}^{2}-v_{x x x x} .
\end{aligned}
$$

We now establish a result that is the foundation of our approach and that is the $n$th conservation law of each hierarchy. As we shall show, such a conservation law can be manipulated to solve the entire hierarchy for all values of $n$. To begin, we establish the $n$th conserved vector of hierarchy (2) by the following theorem.

Theorem 1. The $K d V$ hierarchy possesses the conserved vector $T=\left(T^{t}, T^{x}\right)=\left(u,-D_{x}^{-1}\left(D_{x}^{2}-4 u-2 u_{x} D_{x}^{-1}\right)^{n} u_{x}\right)$ along the solutions of equation (2), i.e., a component of the conserved vector admits a recursion operator.

Proof. Suppose the conservation law is

$$
D_{t} T^{t}+D_{x} T^{x}=0
$$

where $T^{t}$ is the conserved density and $T^{x}$ is the conserved flux. Then, from equation (2), we have

$$
\begin{aligned}
u_{t} & +\left(D_{x}^{2}-4 u-2 u_{x} D_{x}^{-1}\right)^{n} u_{x} \\
& =D_{t}(u)+D_{x}\left(-D_{x}^{-1}\left(D_{x}^{2}-4 u-2 u_{x} D_{x}^{-1}\right)^{n} u_{x}\right) \\
& =0,
\end{aligned}
$$

along the solutions of equation (2), and the result follows. 
Hence, the conserved density for every member of the hierarchy is $T^{t}=u$, while the conserved flux is $T^{x}=-D_{x}^{-1}\left(D_{x}^{2}-4 u-2 u_{x} D_{x}^{-1}\right)^{n} u_{x}$. The fluxes for the first few members of hierarchy (18) for $n=1$ and (21) for $n=2$ are confirmed by the above theorem.

Similarly, we can prove a result for the $\mathrm{mKdV}$ hierarchy.

Theorem 2. The $m K d V$ hierarchy possesses the conserved vector $T=\left(T^{t}, T^{x}\right)=\left(v,-D_{x}^{-1}\left(D_{x}^{2}-4 v^{2}-4 v_{x} D_{x}^{-1} v\right)^{n} v_{x}\right)$ along the solutions of equation (5), i.e., a component of the conserved vector admits a recursion operator.

One can easily check that the conserved vectors (24) for $n=1$ and (26) for $n=2$ arise from this theorem.

In the next section, we give a method to find solutions of the entire hierarchy.

\section{A Method to Solve the Full Hierarchy-Type I Solutions}

To proceed, we require a symmetry generator $X$ to be associated with a conserved vector $T$ of a given equation. Based on the previous section, (14), and (17), we notice that the symmetries $X_{1}$ and $X_{2}$ are possessed by the respective hierarchies for all $n$. In particular, we observe that the point symmetry $\partial_{t}$, when applied to condition (11), is

$$
\partial_{t}\left(u-D_{x}^{-1}\left(D_{x}^{2}-4 u-2 u_{x} D_{x}^{-1}\right)^{n} u_{x}\right)
$$

and for the second symmetry,

$$
\partial_{x}\left(u-D_{x}^{-1}\left(D_{x}^{2}-4 u-2 u_{x} D_{x}^{-1}\right)^{n} u_{x}\right)
$$

Therefore, both symmetries satisfy the association condition, and we conclude that they are associated with every conservation law of Theorem 1, i.e., with any of the $n$th conservation law of the KdV hierarchy member. A similar result holds for the mKdV hierarchy, and here, we conclude that the same symmetries are associated with every conservation law of Theorem 2.

Next, we recall the fundamental theorem on double reduction $[24,25]$, which states that there exist functions $T^{r}$ such that

$$
D_{r} T^{r}=0
$$

The transformed conserved quantity may be expressed as

$$
T^{r}=\frac{T^{t} D_{t}(r)+T^{x} D_{x}(r)}{D_{t}(r) D_{x}(s)-D_{x}(r) D_{t}(s)},
$$

where $r$ and $s$ are similarity variables connected to an associated symmetry $X$.

Since $X_{1}$ and $X_{2}$ are associated with the conserved vector $T$, we consider the linear combination $X=X_{2}+c X_{1}$ ( $c$ is a constant) to obtain the similarity transformation

$$
\begin{aligned}
r & =c x-t, \\
s & =x, \\
u(x, t) & =u(r),
\end{aligned}
$$

for the KdV hierarchy, and similarly,

$$
\begin{aligned}
r & =c x-t, \\
s & =x, \\
v(x, t) & =v(r),
\end{aligned}
$$

for the mKdV hierarchy.

Therefore, we may establish the following results for $T^{r}$.

Theorem 3. The conserved quantity of $K d V$ hierarchy equation (2) can be reduced to

$$
u+c\left(D_{r}^{-1}\left(c^{2} D_{r}^{2}-4 u-2 u_{r} D_{r}^{-1}\right)^{n} u_{r}\right)
$$

where $u=u(r)$.

Proof. Application of (32) gives us

$$
T^{r}=\frac{u(-1)-D_{x}^{-1}\left(D_{x}^{2}-4 u-2 u_{x} D_{x}^{-1}\right)^{n} u_{x} c}{-1}
$$

and in the new variables, by transformation (33), equation (36) transforms to

$$
T^{r}=u+c\left(D_{r}^{-1}\left(c^{2} D_{r}^{2}-4 u-2 u_{r} D_{r}^{-1}\right)^{n} u_{r}\right) .
$$

As examples, $T^{r}$ corresponding to $T_{1}$ of (18) is given by

$$
T^{r}=u_{r r} \cdot c^{3}-3 u^{2} c+u,
$$

for $n=1$, and $T^{r}$ for (21) in the case of $n=2$ is

$$
T^{r}=u_{r r r r} \cdot c^{5}-10 u u_{r r} \cdot c^{3}-5 u_{r}^{2} \cdot c^{3}+10 u^{3} \cdot c+u
$$

Theorem 4. The conserved quantity of $m K d V$ hierarchy equation (5) can be reduced to

$$
v+c\left(D_{r}^{-1}\left(c^{2} D_{r}^{2}-4 v^{2}-4 v_{r} D_{r}^{-1} v\right)^{n} v_{r}\right)
$$

where $v=v(r)$, and the proof is similar to that of Theorem 3 .

Also, to this end, examples of $T^{r}$ for the $m K d V$ hierarchy include $T_{2}$ of (24) given by

$$
T^{r}=v-2 v^{3} \cdot c+v_{r r} \cdot c^{3},
$$

for $n=1$, and $T^{r}$ for $T_{2}$ of (26) is given by

$T^{r}=v+6 v^{5} \cdot c-10 v^{2} v_{r r} \cdot c^{3}-10 v^{2} v_{r}^{2} \cdot c^{3}+v_{r r r} \cdot c^{5}$,

for $n=2$.

That is, the above results can be used to find $T^{r}$ for any value of $n$, for both $K d V$ and $m K d V$ hierarchies. Based on equation (31), we have that $T^{r}=\kappa, \kappa$ is a constant. Therefore, we have reduced the entire partial differential $K d V$ and $m K d V$ hierarchies to ordinary differential hierarchies. These ordinary differential hierarchies may then be solved for any $n$.

\section{Type I Solutions}

In this section, we illustrate the applicability of the above method and theory in establishing solutions to members of the $\mathrm{KdV}$ and $\mathrm{mKdV}$ hierarchy. The solutions obtainable via 
our method in Section 4 will be referred to as type I solutions. Below, we set $\kappa=0$ for simplicity.

5.1. The KdV Hierarchy. Let us consider $n=1$ in Theorem 3 to get the reduced conserved component (38); that is, we solve

$$
u_{r r} \cdot c^{3}-3 u^{2} c+u=0
$$

We find that this equation has an implicit solution

$$
\pm \int^{u(r)} c^{2} \frac{1}{\sqrt{c\left(C_{1} c^{3}+2 a^{3} c-a^{2}\right)}} d a-r-C_{2}=0 .
$$

Suppose we choose the free parameters to be $C_{1}=1, C_{2}=0$, an $\mathrm{d} c=(1 / 2)$; then, the integral in (44) is evaluated to be

$$
\pm \frac{\sqrt{2}}{2 \sqrt{\sqrt{5}-1}} \sqrt{-2 u+1} \sqrt{-4 u+\sqrt{5}+1} \sqrt{4 u+\sqrt{5}-1}
$$

EllipticF $\left(\frac{\sqrt{2}}{\sqrt{\sqrt{5}+1}} \sqrt{-2 u+1}, \frac{2 i}{\sqrt{5}-1}\right) \frac{1}{\sqrt{8 u^{3}-8 u^{2}+1}}$,

where EllipticF is the incomplete elliptic integral of the first kind, and the solution to (43) becomes

$$
\begin{aligned}
u(r)= & \frac{-2 \sqrt{5}-2}{8}\left(\operatorname{JacobiSN}\left(\frac{\sqrt{2 \sqrt{5}-2} r}{2}, \frac{i}{2}+\frac{i}{2} \sqrt{5}\right)\right)^{2} \\
& -\frac{\sqrt{5}}{4}+\frac{\sqrt{-10+10 \sqrt{5}}}{8}+\frac{1}{2}
\end{aligned}
$$

or in the original independent variables, by reversing transformation (33),

$$
\begin{aligned}
u(x, t)= & \frac{-2 \sqrt{5}-2}{8} \\
& \times\left(\operatorname{JacobiSN}\left(\frac{\sqrt{2 \sqrt{5}-2}(c x-t)}{2}, \frac{i}{2}+\frac{i}{2} \sqrt{5}\right)\right)^{2} \\
& -\frac{\sqrt{5}}{4}+\frac{\sqrt{-10+10 \sqrt{5}}}{8}+\frac{1}{2}
\end{aligned}
$$

where JacobiSN is an inverse of elliptic integrals and doubly periodic elliptic functions. These solutions appear graphically in Figure 1.

Next, we consider the second member of the hierarchy, $n=2$, in Theorem 3 to get the reduced conserved component (39). That is, we solve

$$
u_{r r r r} \cdot c^{5}-10 u u_{r r} \cdot c^{3}-5 u_{r}^{2} \cdot c^{3}+10 u^{3} \cdot c+u=0 .
$$

In this case, we find two solutions of the second member of the hierarchy, viz.

$u(x, t)=-\frac{i \operatorname{sech}^{2}\left(-C_{1}+\left(((1 / 2)+(i / 2))(c x-t) / \sqrt{2} c^{5 / 4}\right)\right)}{2 \sqrt{c}}$,

or secondly,
5.2. The $m K d V$ Hierarchy. This time, let us consider $n=1$ in Theorem 4 to get the reduced conserved component (41); that is, we solve

$$
v-2 v^{3} \cdot c+v_{r r} \cdot c^{3}=0 .
$$

The solution of (51) is of two cases, namely,

$$
\begin{aligned}
v(r)= & \pm 2 i \sqrt{2} c^{3} C_{1} \sqrt{-\frac{c}{1-\sqrt{1-4 c^{4} C_{1}}}} \\
& \times \frac{\operatorname{JacobiSN}\left(\sqrt{2} \sqrt{\left(c C_{1} r^{2} / 1-\sqrt{1-4 c^{4} C_{1}}\right)+\left(2 c C_{1} C_{2} r / 1-\sqrt{1-4 c^{4} C_{1}}\right)+\left(c C_{1} C_{2}^{2} / 1-\sqrt{1-4 c^{4} C_{1}}\right)} \mid\left(1-\sqrt{\left.1-4 c^{4} C_{1} / \sqrt{1-4 c^{4} C_{1}}+1\right)}\right)\right.}{1+\sqrt{1-4 c^{4} C_{1}}},
\end{aligned}
$$



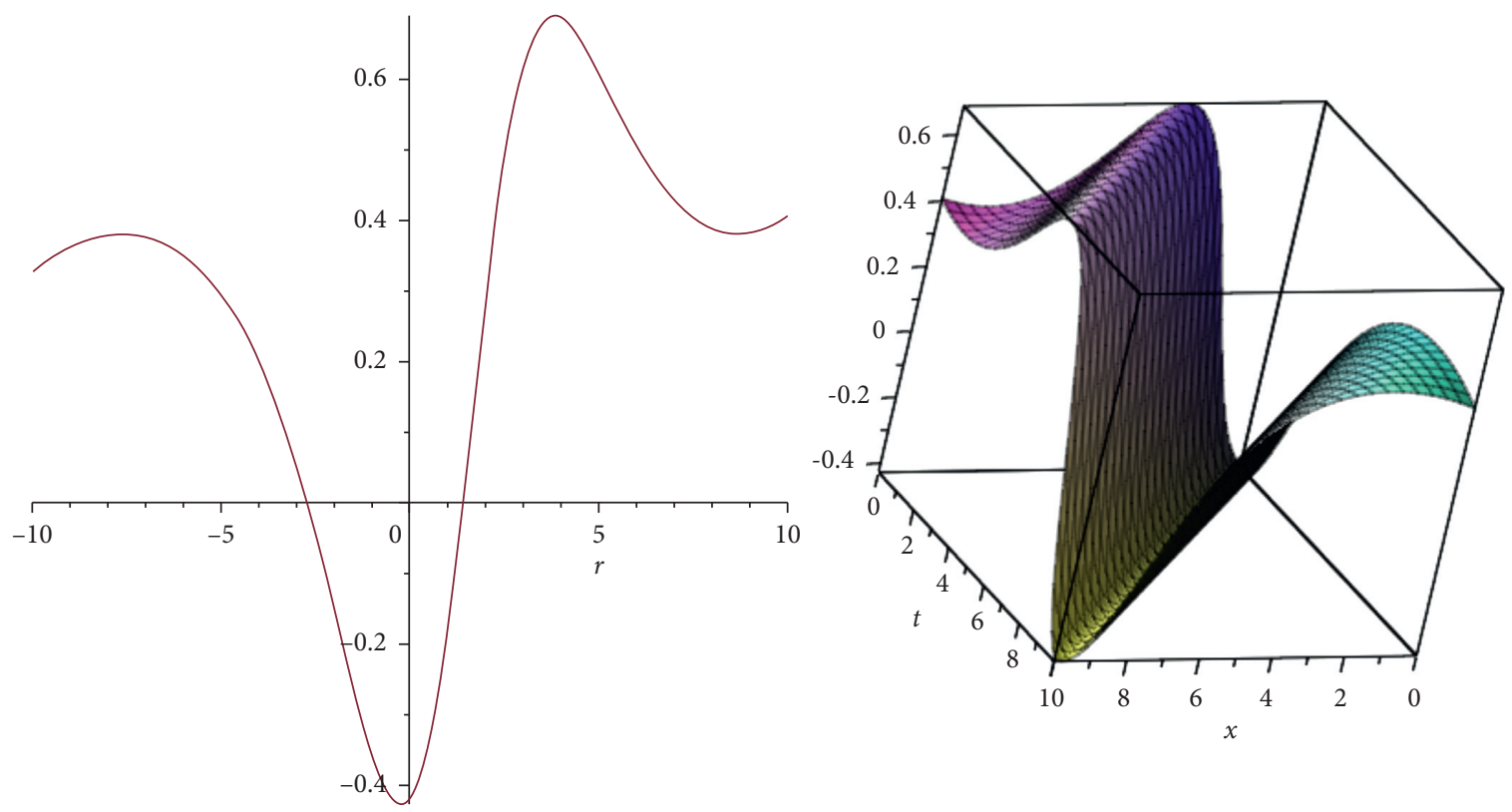

Figure 1: 2D and 3D evolutions of solutions from (46) and (47), respectively.

and secondly,

$$
\begin{aligned}
v(r)= & \frac{C_{2}}{\sqrt{c C_{2}^{2}-c+1}} \text { JacobiSN } \\
& \cdot\left(\frac{\left(r / c^{2}\right) \sqrt{-c(c-1)}+C_{1}}{\sqrt{c C_{2}^{2}-c+1}}, \frac{C_{2}}{c-1} \sqrt{-c(c-1)}\right) .
\end{aligned}
$$

The latter may be expressed in original variables as

$$
\begin{aligned}
v(x, t)= & \frac{C_{2}}{\sqrt{c C_{2}^{2}-c+1}} \text { JacobiSN } \\
& \cdot\left(\frac{\left((c x-t) / c^{2}\right) \sqrt{-c(c-1)}+C_{1}}{\sqrt{c C_{2}^{2}-c+1}}, \frac{C_{2}}{c-1} \sqrt{-c(c-1)}\right) .
\end{aligned}
$$

This solution has 2D and 3D plots in Figure 2.

\section{Type II Solutions}

As seen above, both hierarchies admit conservation laws, such as (19) or (22), independent of Theorems 1 and 2. Now, we cannot transcribe these conservation laws to theorems with a recursion operator as was done in Theorems 1 and 2 . Nonetheless, a $T^{r}$ function may still be obtained in such cases, using the same formula (32) and transformation (33) or (35). This will lead to other solutions, which we call type II.

For example, in the KdV hierarchy, $T^{r}$ for $T_{2}$ of (19) is given by

$$
T^{r}=u u_{r r} \cdot c^{3}-\frac{1}{2} u_{r}^{2} \cdot c^{3}-2 u^{3} c+\frac{u^{2}}{2},
$$

for $n=1$, and $T^{r}$ for $T_{2}$ of (22) is given by

$$
\begin{aligned}
T^{r}= & \frac{15}{2} u^{4} \cdot c-10 u u_{r r} \cdot c^{3}+u u_{r r r} \cdot c^{4}-u_{r} u_{r r r} \cdot c^{5} \\
& +\frac{1}{2} u_{r r}^{2} \cdot c^{5}+\frac{u^{2}}{2},
\end{aligned}
$$

for $n=2$.

As for the $\mathrm{mKdV}$ hierarchy, for $n=1$, we have $T^{r}$ for $T_{1}$ of (23) which is given by

$$
T^{r}=\frac{1}{2} v^{2}+v v_{r r} \cdot c^{3}-\frac{1}{2} v_{r}^{2} \cdot c^{3}-\frac{3}{2} v^{4} \cdot c,
$$

and $T^{r}$ for $T_{1}$ of (25) is given by

$$
T^{r}=\frac{1}{2} v^{2}+5 v^{6} \cdot c-10 v^{3} v_{r r} \cdot c^{3}-5 v^{2} v_{r r}^{2} \cdot c^{5}
$$

$$
+v v_{r r r r} \cdot c^{5}-v_{r} v_{r r r} \cdot c^{5}+\frac{1}{2} v_{r r}^{2} \cdot c^{5}
$$

for $n=2$. Below, we explore some solutions that arise out of these $T^{r}$ functions.

6.1. Type II Solution to the KdV Hierarchy. A type II solution corresponding to solving (55) yields an implicit solution

$$
\pm \int^{u(r)} c^{2} \frac{1}{\sqrt{c a\left(C_{1} c^{3}+2 a^{2} c-a\right)}} d a-r-C_{2}=0 .
$$

Here, the above integral is equal to 



Figure 2: Evolution of solutions corresponding to (53) and (54), respectively.

$$
\begin{aligned}
& \frac{1}{2} \frac{c\left(-1+\sqrt{-8 C_{1} c^{4}+1}\right) \sqrt{2}}{\sqrt{c u(r)\left(C_{1} c^{3}+2(u(r))^{2} c-u(r)\right)}} \times \sqrt{\frac{4 c u(r)+\sqrt{-8 C_{1} c^{4}+1}-1}{-1+\sqrt{-8 C_{1} c^{4}+1}} \times \sqrt{\frac{-4 c u(r)+\sqrt{-8 C_{1} c^{4}+1}+1}{\sqrt{-8 C_{1} c^{4}+1}}}} \\
& \quad \times \sqrt{-\frac{c u(r)}{-1+\sqrt{-8 C_{1} c^{4}+1}} \times \text { EllipticF }\left(\sqrt{\frac{4 c u(r)+\sqrt{-8 C_{1} c^{4}+1}-1}{-1+\sqrt{-8 C_{1} c^{4}+1}}}, \frac{1}{2 \sqrt{2}} \sqrt{\frac{-1+\sqrt{-8 C_{1} c^{4}+1}}{\sqrt{-8 C_{1} c^{4}+1}}}\right)} .
\end{aligned}
$$

Suppose we let $C_{1}=1, C_{2}=0$, and $c=(1 / 2)$; then, the explicit solution to (59) is

$$
\begin{aligned}
u(r)= & \frac{(-2+\sqrt{2})}{4} \\
& \times\left(\left(\operatorname{JacobiSN}\left(r \sqrt[4]{2}, \frac{i}{2} \sqrt{2-\sqrt{2}} \sqrt[4]{2}\right)\right)^{2}-1\right) .
\end{aligned}
$$

or in original variables,

$$
u(x, t)=\frac{(-2+\sqrt{2})}{4} \times\left(\left(\operatorname{JacobiSN}\left((c x-t) \sqrt[4]{2}, \frac{i}{2} \sqrt{2-\sqrt{2}} \sqrt[4]{2}\right)\right)^{2}-1\right)
$$

The progression of these solutions appears in Figure 3, and they are visibly periodic in nature.
6.2. Type II Solution to the $m K d V$ Hierarchy. A type II solution corresponding to solving (57) has an implicit solution 

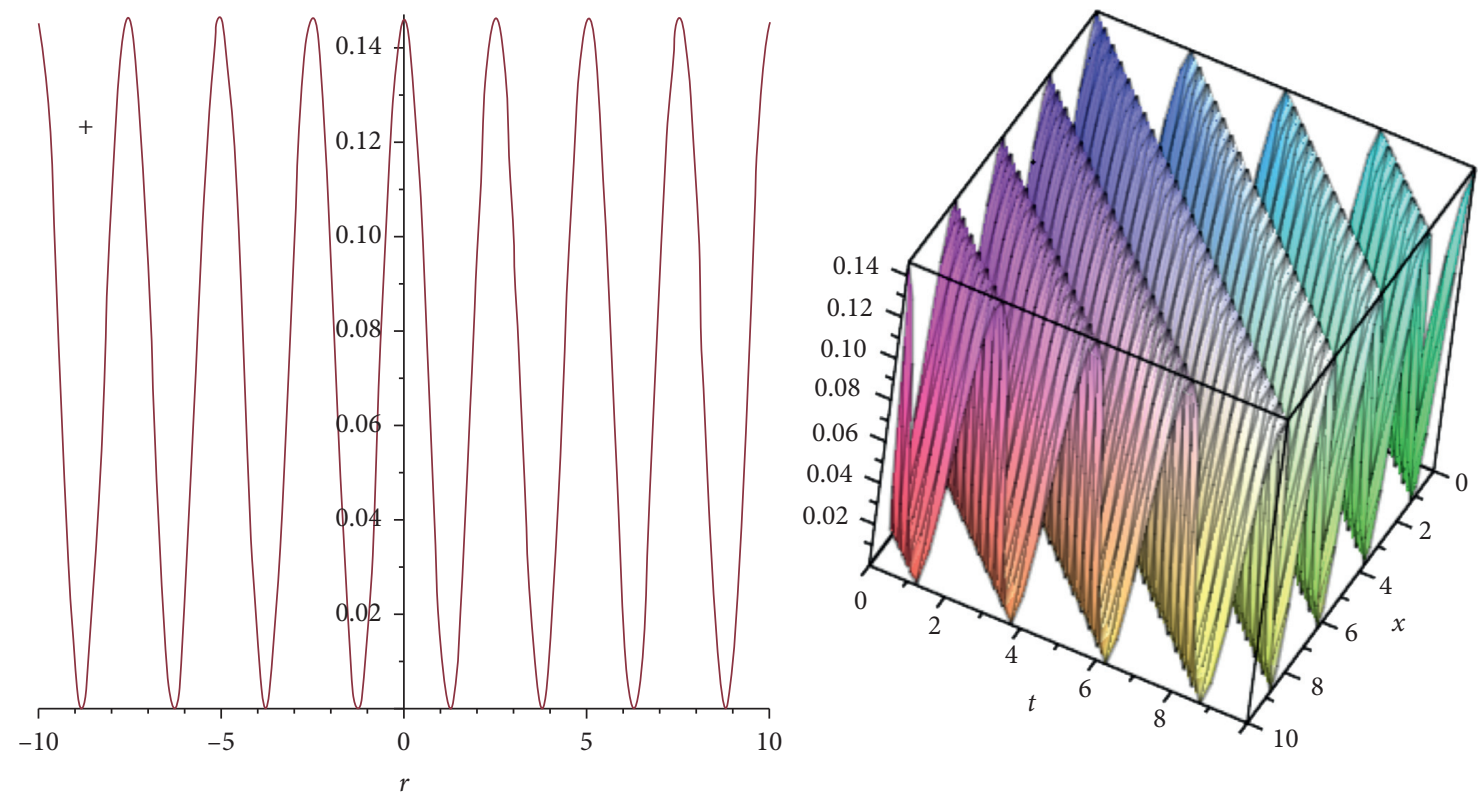

Figure 3: 2D and 3D progression of solutions corresponding to (61) and (62), respectively.
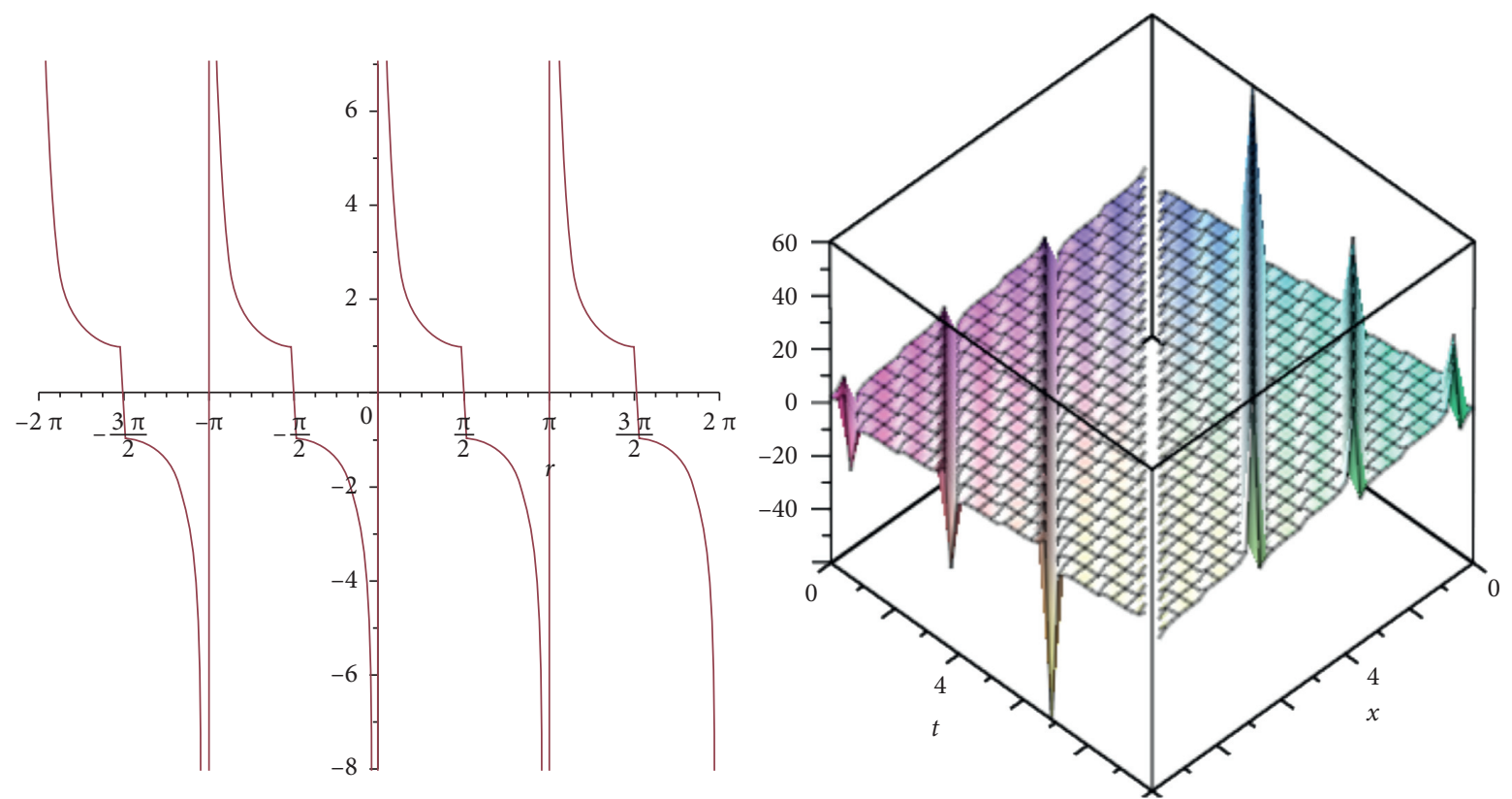

FIGURE 4: 2D and 3D plots corresponding to (65) and (66), respectively. 


$$
\pm \int^{v(r)} c^{2} \frac{1}{\sqrt{c a\left(C_{1} c^{3}+a^{3} c-a\right)}} d a-r-C_{2}=0
$$

The integral is evaluated to be

$$
\begin{gathered}
\frac{1}{2} \frac{c\left(-1+\sqrt{-8 C_{1} c^{4}+1}\right) \sqrt{2}}{\sqrt{c v(r)\left(C_{1} c^{3}+2(v(r))^{2} c-v(r)\right)}} \times \sqrt{\frac{4 c v(r)+\sqrt{-8 C_{1} c^{4}+1}-1}{-1+\sqrt{-8 C_{1} c^{4}+1}}} \times \sqrt{\frac{-4 c v(r)+\sqrt{-8 C_{1} c^{4}+1}+1}{\sqrt{-8 C_{1} c^{4}+1}}} \\
\times \sqrt{-\frac{c v(r)}{-1+\sqrt{-8 C_{1} c^{4}+1}}} \times \operatorname{EllipticF}\left(\sqrt{\frac{4 c v(r)+\sqrt{-8 C_{1} c^{4}+1}}{-1+\sqrt{-8 C_{1} c^{4}+1}}}, \frac{1}{2 \sqrt{2}} \sqrt{\frac{-1+\sqrt{-8 C_{1} c^{4}+1}}{\sqrt{-8 C_{1} c^{4}+1}}}\right) .
\end{gathered}
$$

Suppose we let $C_{1}=1, C_{2}=0$, and $c=(1 / 2)$; then, the solution to $(63)$ is

$$
v(r)= \pm \frac{1}{\tan (r)} \sqrt{(\tan (r))^{2}+1},
$$

or

$$
v(x, t)= \pm \csc (c x-t),
$$

whose graphical representation appears in Figure 4.

\section{Conclusions}

In the study of differential equations, equations that are highly nonlinear and that possess higher-order derivatives are almost impossible to solve. We have proposed a scheme to overcome this problem and aid the solution of, in particular, the KdV and $\mathrm{mKdV}$ infinite hierarchy.

The well-known (solitary wave) solution of the $\mathrm{KdV}$ equation involves the hyperbolic secant function [26], but Korteweg and de Vries were interested in cnoidal solutions, expressible in terms of Jacobi's elliptic CN functions [27]. Given the mathematical relations between the Jacobi SN and $\mathrm{CN}$ solutions, sin, cos, sech, and tanh functions, our above solutions for $\mathrm{KdV} n=1$ may be related to the known ones, but in that case, the recovery of known or related solutions validates our approach. As for our solutions for $\operatorname{KdV} n=2$, we find no connection to any known results. It is possible to find many more solutions.

In the analysis of our solutions, we divided our solutions to be of two types: type I and type II. Type I is the most interesting solution as it is derived from a recursion operator within the conservation law of the $\mathrm{KdV}$ and $\mathrm{mKdV}$ infinite hierarchy. In both solution types, the knowledge of association between symmetry and conserved components was exploited and formed the basis of our approach to reduce the order of the partial differential hierarchy to an ordinary differential hierarchy. Consequently, our method has many significant uses and can be extended to solve other infinite hierarchies. Specifically, it may be applied to any hierarchy in possession of a recursion operator, for example, the Kaup-Kupershmidt hierarchy. Furthermore, it would be interesting to attempt a study of systems of nonlinear equations with known recursion operators, such as the Hirota-Satsuma system or the nonlinear Schrödinger system of real equations.

An advantage of our approach is that it can easily be implemented into computer algebra programs such as Maple or Mathematica. A disadvantage is that, at higherorder members of the hierarchy, one may struggle to solve the reduced conservation laws, simply because the computations are too involved and computer algebra programs may run out of memory to complete the necessary calculations.

In the known literature, there are numerous methods to solve members of the KdV hierarchy, for example, the generalized Kudryashov method [28], the double Laplace transform [29, 30], the differential transform method [31], the tanh-expansion method [32], the exp-function method [33], and the $G^{\prime} / G$-expansion method [34]. Our approach involves a recursion operator and conservation law to aid the analysis of the nonlinear partial differential hierarchy. To the best of our knowledge, this is the first time that a study has conceived an approach for dealing with the entire $\mathrm{KdV}$ hierarchy.

\section{Data Availability}

No data were used to support this study.

\section{Conflicts of Interest}

The authors declare no conflicts of interest.

\section{Acknowledgments}

This work received financial support from the National Research Foundation of South Africa (118047).

\section{References}

[1] E. M. De Jager, "On the origin of the Korteweg-de Vries equation," Forum der Berliner Mathematischen Gesellschaft, vol. 19, pp. 171-195, 2011.

[2] J. W. Miles, "The Korteweg-de Vries equation: a historical essay," Journal of Fluid Mechanics, vol. 106, no. 1, pp. 131-147, 1981. 
[3] X. Tian, 2015 A summary of the Korteweg-De Vries equation.

[4] I. Avramidi and R. Schimming, "A new explicit expression for the Korteweg-de Vries hierarchy," Mathematische Nachrichten, vol. 219, no. 1, pp. 45-64, 2000.

[5] T. L. Perelman, A. Fridman, and M. M. Eliashevich, "Modified Korteweg-de Vries equation in electro hydrodynamics," Zhurnal Eksperimental'noi i Teoreticheskoi Fiziki, vol. 66, pp. 1316-1323, 1974.

[6] H. Schamel, "A modified Korteweg-de Vries equation for ion acoustic wavess due to resonant electrons," Journal of Plasma Physics, vol. 9, no. 3, pp. 377-387, 1973.

[7] U. Obaidullah and S. Jamal, "A computational procedure for exact solutions of Burgers' hierarchy of nonlinear partial differential equations," Journal of Applied Mathematics and Computing, vol. 65, no. 1-2, pp. 541-551, 2021.

[8] U. Obaidullah and S. Jamal, "On the formulaic solution of a $(n+1)$ th order differential equation," International Journal of Algorithms, Computing and Mathematics, vol. 7, no. 3, p. 58, 2021.

[9] R. M. Miura, C. S. Gardner, and M. D. Kruskal, "Korteweg-de Vries equation and generalizations. II. Existence of conservation laws and constants of motion," Journal of Mathematical Physics, vol. 9, no. 8, pp. 1204-1209, 1968.

[10] P. J. Olver, "Evolution equations possessing infinitely many symmetries," Journal of Mathematical Physics, vol. 18, no. 6, pp. 1212-1215, 1977.

[11] M. J. Ablowitz, A. Ramani, and H. Segur, "A connection between nonlinear evolution equations and ordinary differential equations of P-type. I," Journal of Mathematical Physics, vol. 21, no. 4, pp. 715-721, 1980.

[12] M. J. Ablowitz and P. A. Clarkson, "Solitons, nonlinear evolution qquations and inverse scattering," in London Mathematical Society Lecture Note Series Vol. 149, Cambridge University Press, Cambridge, England, 1991.

[13] M. J. Ablowitz and H. Segur, Solitons and the Inverse Scattering Transform, SIAM Studies in Applied Mathematics, Philadelphia, PA, USA, 1981.

[14] C. S. Gardner, J. M. Greene, M. D. Kruskal, and R. M. Miura, "Method for solving the Korteweg-deVries equation," Physical Review Letters, vol. 19, no. 19, pp. 1095-1097, 1967.

[15] R. Hirota, "Direct methods in soliton theory," in Solitons, R. Bullough and P. Caudrey, Eds., vol. 17, New York, NY, USA, Springer-Verlag, 1980.

[16] G. A. Guthrie, Constructing Miura Transformations Using Symmetry Groups, University of Canterbury, Christchurch, New Zealand, 1993.

[17] R. A. Kraenkel, M. A. Manna, and J. G. Pereira, "The Korteweg-de Vries hierarchy and long water-waves," Journal of Mathematical Physics, vol. 36, no. 1, pp. 307-320, 1995.

[18] P. J. Olver, Application of Lie Groups to Differential Equations, Springer, New York, NY, USA, 1993.

[19] A. H. Kara and F. M. Mahomed, "The relationship between symmetries and conservation laws," International Journal of Theoretical Physics, vol. 39, no. 1, pp. 23-40, 2000.

[20] I. M. Gel'fand and S. V. Fomin, Calculus of Variations, Prentice-Hall, Englewood Cliffs, NJ, USA, 1963.

[21] H. Steudel, "Über die Zuordnung zwischen lnvarianzeigenschaften und Erhaltungssätzen," Zeitschrift für Naturforschung A, vol. 17, no. 2, pp. 129-132, 1962.

[22] G. B. Whitam, "Nonlinear dispersive waves," Proceedings of the Royal Society of Series A, vol. 283, pp. 238-261, 1965.

[23] N. Zabusky, "A synergetic approach to problems of nonlinear dispersive wave propagation and interaction," in Nonlinear
Partial Differential Equations, F. Ames, Ed., Academic Press, New York, NY, USA, 1967.

[24] A. Sjöberg, "Double reduction of PDEs from the association of symmetries with conservation laws with applications," Applied Mathematics and Computation, vol. 184, Article ID 608616, 2007.

[25] A. H. Bokhari, A. Y. Al-Dweik, F. D. Zaman, A. H. Kara, and F. M. Mahomed, "Generalization of the double reduction theory," Nonlinear Analysis: Real World Applications, vol. 11, no. 5, pp. 3763-3769, 2010.

[26] J. S. Russel, "Report on Waves," Report of the 14th Meeting of the British Association For the Advancement of Science, John Murray, London, UK, 1844.

[27] P. G. Drazin and R. S. Johnson, Solitons: An Introduction, Cambridge University Press, Cambridge, England, 1989.

[28] N. A. Kudryashov, "On types of nonlinear nonintegrable equations with exact solutions," Physics Letters A, vol. 155, no. 4-5, pp. 269-275, 1991.

[29] T. A. Estrin and T. J. Higgins, "The solution of boundary value problems by multiple Laplace transformations," Journal of the Franklin Institute, vol. 252, no. 2, pp. 153-167, 1951.

[30] L. Debnath, "The double Laplace transforms and their properties with applications to functional, integral and partial differential equations," International Journal of Algorithms, Computing and Mathematics, vol. 2, no. 2, pp. 223-241, 2016.

[31] K. Zhou, Differential Transformation and its Application for Electrical Circuits, Huarjung University Press, Wuuhahn, China, 1986, in Chinese.

[32] W. Malfliet and W. Hereman, "The tanh method: I. Exact solutions of nonlinear evolution and wave equations," Physica Scripta, vol. 54, no. 6, pp. 563-568, 1996.

[33] J.-H. He and X.-H. Wu, "Exp-function method for nonlinear wave equations," Chaos, Solitons \& Fractals, vol. 30, no. 3, pp. 700-708, 2006.

[34] M. Wang, X. Li, and J. Zhang, "The $G^{\prime} / G$-expansion method and travelling wave solutions of nonlinear evolution equations in mathematical physics," Physics Letters A, vol. 372, no. 4 , pp. 417-423, 2008. 